\title{
ZUM PROBLEM DES DENUNZIANTEN. EINIGE LITERARISCHE UND RECHTSTHEORETISCHE ÜBERLEGUNGEN
}

Unter der Überschrift „Haben Sie niemand zu verdächtigen?“ wurde im November 1937 in der Zeitung der Schutzstaffeln (SS) der NSDAP, „Das Schwarze Korps“, darauf hingewiesen, daß es bei bösem Willen nicht schwer sei, jemanden im Verdacht zu haben. Irgendwelche Umstände, die Mißtrauen hervorrufen könnten, seien immer zu finden. Viel schwieriger sei es dagegen, so das „Schwarze Korps“, ein solches Mißtrauen wieder zu beseitigen. Deshalb solle es sich jeder gut überlegen, bevor er einen Verdacht ausspreche, der zu einer Anzeige führen würde. ${ }^{1}$

Die Ambivalenz von politischen Denunziationen bildete ein charakteristisches Merkmal der nationalsozialistischen Herrschaftsordnung. Einerseits wurde die Bevölkerung zu Anzeigen aufgefordert, andererseits aber das Denunziantentum bekämpft. In der Öffentlichkeit bezeugte man die moralische Ächtung der Denunziation und des Denunzianten. Gleichzeitig aber gab es eine weitgehende Legitimierung und Nutzung von Denunziationen. Irmgard Keun schrieb in den Bildern und Gedichten aus der Emigration:

Immer noch zögerte ich, nun selbst mit Schreiben anzufangen und für mein Buch ein Deutschland der Nationalsozialisten lebendig werden zu lassen [...]. Ein Deutschland voll berauschter Spießbürger. Berauscht, weil sie es sein sollten - berauscht, weil man ihnen Vernunftlosigkeit als Tugend pries -, berauscht, weil sie gehorchen und Angst haben durften, und berauscht, weil sie Macht bekommen haben. Genügte nicht ein Gang zur Gestapo, um sämtliche Stammtischgenossen zumindest ein bißchen unter Verfolgung zu setzen? ${ }^{2}$

${ }^{1}$ Vgl. „Das Schwarze Korps“. Zeitung der Schutzstaffeln der NSDAP. Organ der Reichsführung SS, 3. Jg., 46. Folge, 18. 11. 1937. In: Gisela Diewald-Kerkmann: Politische Denunziation im NSRegime oder die kleine Macht der „Volksgenossen“. Bonn 1995, S. 137.

${ }^{2}$ Irmgard Ke un: Bilder und Gedichte aus der Emigration. Köln 1947. In: Irmgard Ke un: Nach Mitternacht. Hrsg. v. Dietrich Stein bach. Stuttgart 1982, S. 163. 
In dem in der Zeit zwischen 1935 und 1937 im Exil geschriebenen Roman Nach Mitternacht schildert Irmgard Keun das alltägliche Leben in Deutschland nach 1933. Beinahe in jeder Szene des Romans konfrontiert sie uns mit dem Phänomen des unter NS-Herrschaft weitgehend legitimierten Denunziantentums. Ihre Protagonisten scheinen allerdings besonders in Wirtshäusern und Cafés der Gefahr des Angezeigtwerdens ausgeliefert zu sein. In den ersten Jahren der Existenz des Dritten Reiches bildete in einer großen Zahl der Fälle tatsächlich eben das Wirtshaus, so Karol Sauerland, den Ausgangspunkt für Denunziationen. Viele konnten sich einfach nicht daran gewöhnen, daß öffentliche Räume für den politischen Meinungsaustausch nicht mehr geeignet waren. ${ }^{3}$

Sanna und Gerti, zwei befreundete Mädchen im Roman Nach Mitternacht, verkehren weiterhin in den Lokalen. Dort verbringen sie einen Abend zusammen mit dem jungen SA-Mann Kurt Pielmann und einem Herrn Kulmbach, der ebenfalls ein überzeugter Nationalsozialist ist.

Ausgerechnet vor diesem [...] Kulmbach stänkert Gerti den Kurt Pielmann auf die gefährlichste Weise an. [...] Sie sagt, der Führer habe doch mal erklärt, daß die Juden alle nach Knoblauch riechen. Sie möchte nun bloß mal wissen, an wieviel Juden der Führer schon gerochen habe. [...] Die Juden, die sie kenne, würden jedenfalls nicht riechen, und was Knoblauch anbelangte, so esse sie den sehr gern. Daraufhin Pielmann entsetzlich aufgeregt: Wenn Gerti so sprechen könne, sei sie rassisch verseucht. Kulmbach versucht, Pielmann zu beruhigen, und sagt, er habe auch mal einen anständigen Juden kennengelernt, und bestellt daraufhin noch eine Runde Kirsch. ${ }^{4}$

Glücklicherweise konnte die vorsichtigere Sanna ihre Freundin dazu bewegen, mit ihr auf die Toilette zu gehen, um diese heikle Diskussion abzubrechen. An einem anderen Tage saßen sie wieder einmal in einem Lokal:

Aus dem Lautsprecher rasten Reden wie ein Gewitter. Voll war das Café von diesen Reden über den Führer, [...] über die Begeisterung der Menge. Zwei alte Frauen kamen herein [...]. Als sie anfangen wollten zu essen, wurde im Radio das Horst-Wessel-Lied gespielt, die alten Fräuleins ließen ihre Löffel fallen, standen auf, reckten die Arme. Das muß man, weil man nie weiß, wer einen beobachtet und anzeigt. Vielleicht hatten sie voreinander Angst. ${ }^{5}$

Dieses Verhalten kann nicht überraschen, wenn man bedenkt, daß bei der Aufschlüsselung nach situativen Momenten der "Tatort" Lokal mit weitem Vorsprung den ersten Platz einnimmt. Untersuchungen von Klaus-Michael Mallmann und Gerhard Paul haben darüber hinaus ergeben, daß in $90 \%$ der analysierten Fälle die Anzeigen von anderen Gästen ausgingen, in $10 \%$ vom Wirt oder der Wirtin. Ein Drittel der Denunzierten gaben an, bei ihren Bemerkungen unter Alkoholeinfluß gestanden zu haben. ${ }^{6}$

Eine lebenskluge Frau im Roman Nach Mitternacht erzählte, daß sie ihren Mann nicht mehr in die Wirtschaft gehen ließ:

${ }^{3}$ Karol Sauerland: Dreißig Silberlinge. Denunziation. Gegenwart und Geschichte. Berlin 2000, S. 52.

${ }^{4}$ Keun (wie Anm. 2), S. $28 f$,

${ }^{5}$ Ebenda, S. $22 f$.

${ }^{6}$ Vgl. Sauerland (wie Anm. 3), S. 52 f. 
Eine Frau, die ihren Mann [...] sich erhalten will, läßt ihn in der heutigen Zeit nicht zum Stammtisch. [...] Männer haben en loses Mundwerk, und wenn sie dann was geladen haben, fangen sie an, über die [...] Politik zu quasseln [...] und drecklige Bemerkungen zu machen, und meinen, sie wären unter lauter guten Freunden. Am nächsten Tag sitzen se dann da mit em dicken Kopp, und irgendein Neidiger, dem sein Geschäft gerad nit jod geht, is schon nach der Gestapo [...] am rennen, für en Anzeig zu erstatten.

Denunziationen gab es natürlich nicht nur in öffentlichen Räumen, sondern auch am Wohnort, wo Nachbarn sich schnell in Zuträger verwandeln konnten. Sogar die eigenen vier Wände gewährten keine Sicherheit, vor allem, wenn Familienstreit zum Alltag gehörte.

Die von Strafrechtlern 1934 in bezug auf das „Blutschutzgesetz“ geäußerten Befürchtungen, daß die Denunziationen nicht nur eine erschreckende Zahl erreichen, sondern ,auch ein ganz besonders unerfreuliches, ja widerwärtiges Gesicht annehmen “8 würden, bestätigten sich. Aus Feindschaft oder aus purer Gehässigkeit wurden Nachbarn, Arbeitskollegen oder Vorgesetzte angezeigt und beschuldigt. Meistens handelte es sich um Konflikte - so ermittelte Gisela DiewaldKerkmann -, die schon über einen längeren Zeitraum unter Verwandten, Nachbarn, Arbeitskollegen, Bekannten oder zwischen Vorgesetzten und Untergebenen, Vermietern oder Mietern bestanden und sich in der politischen Denunziation entluden. ${ }^{9}$ Die politische Anzeige wurde zu einem gängigen Mittel, das soziale Animositäten, berufliche Rivalitäten oder persönliche Streitigkeiten auszutragen half. Irmgard Keun läßt in ihrem Roman einen alten Mann zur Gestapo gehen und dort erklären:

[...] es handelt sich da um meinen Sohn [...]. Die Frau Fabrizius von der ersten Etage [...] hat Anzeige gegen ihn erstattet, daß er in betrunkenem Zustand die Trepp runterjefallen wär und dabei auf den Herrn Minister Göring geschimpft hätt. [...] es ist nämlich nur, weil Fabrizius mit meiner Schwiegertochter immer im Streit liegt. ${ }^{10}$

Bei derselben Gestapo-Stelle erschien eine uralte Frau, die stundenlang ,von ihrem Untermieter, der keine Miete zahle und Kommunist“ sei, erzählte. „Er habe die Hakenkreuzfähnchen, mit denen sie den Balkon geschmückt hatte, abgerissen. Nein, gesehen habe sie es nicht [...]. Wieso er denn Kommunist sei? Ja, durch sein ganzes Verhalten. “11

Sanna, die dies zu hören bekam, weil sie am selben Tag von der Gestapo abgeholt wurde, beobachtet, wie immer mehr Menschen herbeiströmen. Das Gestapo-Zimmer „scheint die reinste Wallfahrtstätte. Mütter zeigen ihre Schwiegertöchter an, Töchter ihre Schwiegerväter, Brüder ihre Schwestern, Schwestern ihre

\footnotetext{
${ }^{7}$ Keun (wie Anm. 4), S. 65.
}

${ }^{8}$ Protokoll der Strafrechtskommission, 5. 6. 1934. Zit. nach Diewald-Kerkmann (wie Anm. 1), S. 98 .

${ }^{9}$ Gisela Diewald-Kerkmann: Politische Denunziation im NS-Regime. In: Günter Jerouschek u.a. (Hrsg.): Denunziation. Historische, juristische und psychologische Aspekte. Tübingen 1997, S. $150 \mathrm{f}$.

${ }^{10}$ Keun (wie Anm. 4), S. 60.

${ }^{11}$ Ebenda, S. 59f. 
Brüder, Freunde ihre Freunde, Stammtischgenossen ihre Stammtischgenossen, Nachbarn ihre Nachbarn." "12 Als Sanna dann endlich nach vielen Stunden vernommen wurde, erfuhr sie, daß sie wegen staatsfeindlicher Reden über die Rundfunkansprachen Görings und verächtlich machender Bemerkungen über den Führer angezeigt wurde. Von wem? Von ihrer Tante Adelheid, bei der sie gewohnt und in deren Sohn Franz sie sich verliebt hat. Gegen diese Beziehung versuchte die Tante mit allen Mitteln vorzugehen.

Nach dem Verhör verläßt Sanna fluchtartig die Stadt, um in Frankfurt bei ihrem Bruder zu leben. Inzwischen hatte Franz gemeinsam mit einem Freund einen kleinen Tabakladen gemietet und eingerichtet, um dadurch für Sanna und sich eine von seiner Mutter unabhängige Existenz aufzubauen. Dieses Vorhaben scheitert jedoch, weil ein SA-Mann, dessen Ansehen in der SA gelitten hatte (es gab Gerüchte, seine Großmutter sei Jüdin gewesen), eine Tat begehen wollte, „die ihn bei den Nazis wieder geachtet und beliebt machte, und er wollte den Zigarettenladen von Franz [...] kaputtmachen, in seiner Straße wollte er keine Konkurrenz (er war selbst Besitzer eines Tabakladens)“. ${ }^{13}$ Der SA-Mann ging zur nächsten Parteistelle und zeigte Franz wegen kommunistischer Umtriebe und zersetzender Reden an. Auf diese Weise konnte man damals jeden Konkurrenten schnell loswerden.

$\mathrm{Da}$ es sich bei der politischen Denunziation nicht selten um eine Instrumentalisierung privater Konflikte handelte, war auch den NS-Instanzen bewußt. Aber weder private Motive noch eindeutige Interessen des Anzeigenden hinderten die NSDAP oder die Gestapo daran, dem Signalisieren politisch unkorrekten Handelns nachzugehen. Und selbst, wenn man die Denunzierten später entlassen hat, weil ihnen nichts nachzuweisen war, blieb nichts, wie es früher war. So ist auch das kleine Unternehmen von Franz zugrunde gegangen.

Heini, ein bekannter Journalist im Roman Nach Mitternacht, spricht eine nüchterne und zugleich erschreckende Erkenntnis aus: „Wir leben nun mal in der Zeit der großen deutschen Denunziantenbewegung. Jeder hat jeden zu bewachen, jeder hat Macht über jeden. Jeder kann jeden einsperren lassen. Der Versuchung, diese Macht auszuüben, können nur wenige widerstehen. "14 Die einfachen Menschen haben für einen Augenblick etwas zu sagen, sind in dem Moment Teil eines historischen Ereignisses oder einer großen Bewegung. Und infolge der verkürzten Rechtswege und der Anpassung des Rechts an momentan herrschende Interessen gelangen sie zu schnellem Erfolg: ,[...] sie sind nicht nur Zuschauer, sondern Akteure, wenngleich mit einem kurzen Auftritt“ - stellt Karol Sauerland fest. ${ }^{15}$

Allerdings gab es auch nach 1933 keine gesetzliche Vorschrift, politische Vergehen anderer zu melden. Eine gesetzliche Anzeigepflicht sei schon allein deshalb nicht notwendig, machte der Reichsjustizminister deutlich, da bereits für jeden „Volksgenossen“ die „sittliche Pflicht“ bestehe, „, an der inneren Sicherung

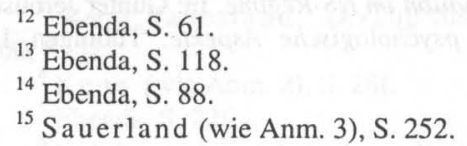


des Reichs und der eigenen kampfbereiten Volksgemeinschaft gegen reichs- und staatsfeindliche Handlungen aktiv mitzuwirken“. ${ }^{16}$ Diese „sittliche Pflicht“ hatte ihr Fundament in der Volksgemeinschaftsideologie und bestand auch ohne gesetzliche Festlegung. Tatsächlich wurde von jedem „Volksgenossen“ erwartet, daß alle „Volksschädlinge“, alle Kritiker des NS-Regimes, „Miesmacher“, „Nörgler" und „Defätisten“ rechtzeitig und rücksichtslos namhaft gemacht werden. Mit dem Aufruf zur Erfüllung der „Treuepflicht gegenüber Führer und Volk“ wollte man nicht nur einen Abbau von Hemmungen und Reaktionen des Gewissens, sondern auch eine Ausschaltung sonst gültiger Norm- und Wertvorstellungen erreichen. Die ausgesprochen hohe Zahl der politischen Denunziationen macht sichtbar, daß erschreckende Grausamkeiten in einem Klima von Normalität verübt werden konnten, weil sie mit „gewissen Formen der Sittlichkeit“, d.h. Erwartungen, Forderungen und Ideologien des Kollektivs im Einklang standen. ${ }^{17}$ Das gewissenhafte Signalisieren jeder Form von Nonkonformität und Dissens konnte sogar ohne weiteres mit sensiblen Gewissensreaktionen im Bereich der Binnenmoral einhergehen. Das durch die Denunzianten immer wieder betonte Moment der Pflichterfüllung oder andersartige Rechtfertigungsversuche bezeugen, daß die „private Moral“" trotz allem intakt blieb.

Der bereits erwähnte Journalist Heini erklärte einmal seinen Stammtischgenossen, warum er in dem „trübseligsten Absteigequartier Frankfurts“ wohnen müsse. Wenn er in einem besseren Hotel wohnen würde,

könnte das Neid erwecken, und Manderscheid oder irgendein anderer würde mich wegen verächtlichmachender Reden über die Regierung denunzieren. [...] Regen Sie sich nicht auf, Manderscheid, ich wollte Sie nicht beleidigen. Was Sie heute noch unterlassen, werden Sie morgen tun. Sie haben Familie. Ein Mann mit Familie wird feige und kann sich heutzutage keinen Charakter leisten. Für viele bildet die Familie auch nur den erwünschten moralischen Vorwand für Schlappheit und Kriecherei. ${ }^{18}$

Der Roman Die Toten bleiben jung von Anna Seghers, der im Jahre 1949 veröffentlicht wurde, stellt einen Versuch dar, die deutsche Zeitgeschichte von 1918 bis 1945 episch zu verarbeiten. Anna Seghers wollte Personen und Handlungen schildern, die typisch für die Gesellschaft jener Zeit waren. Auch in ihrem Roman begegnen wir vielen Denunzianten sowie zahlreichen Situationen einer Anzeigeerstattung, welche die Koexistenz der privaten Moral und der kollektiven „Sittlichkeit“ belegen. Ilse Wenzlow, eine der Gestalten des Romans Die Toten bleiben jung, hat einen Pfarrer angezeigt, weil er als Seelsorger ihrer Kinder im Konfirmandenunterricht „Aussprüche täte, die der Anschauung des nationalsozialistischen Staates zuwider[liefen]“. ${ }^{19}$ Die besorgte Mutter hatte den Fall einfach melden müssen, auch wenn man diesen Schulpfarrer danach für seine

${ }^{16} \mathrm{Vgl}$. das Schreiben des Reichsministers der Justiz (RJM) an den Ministerrat, 13. 10. 1939; BA, R 43 II/1264 a, Bl. 115ff. In: Diewald-Kerkmann (wie Anm. 1), S. 21.

17 Vgl. Herbert Jäger: Makrokriminalität. Studien zur Kriminologie kollektiver Gewalt. Frankfurt (M.) 1989, S. 145.

${ }^{18}$ Keun (wie Anm. 4), S. 88f.

${ }^{19}$ Anna Seghers: Die Toten bleiben jung. Berlin/Weimar 1974, S. 417. 
Vermessenheit zur Verantwortung zog. Auch ,wenn er sogar noch in einem KZ landete, dann war das allein seine eigene Schuld“. ${ }^{20}$ Der Vorfall hatte bei ihr aber eine „Beunruhigung des Gewissens“ zur Folge. „Denn schließlich war Ilse Wenzlow strenge Christin vom Elternhaus her. Da hatte sie nicht einfach gehorchen können, wie es Wenzlow verlangte, sie hatte nachdenken müssen, ob sie tat, was ihr Gewissen vorschrieb. Das hatte sie schlaflose Nächte gekostet, die Vorschriften ihres Gewissens mit denen des Mannes zu vereinen. ${ }^{\text {"21 }}$ Weil sie aber von ihrem Mann, einem überzeugten Nazi-Offizier der Wehrmacht, oft genug Sätze zu hören bekam, wie z.B.: „Man soll in Gegenwart seiner Kinder nie den Staat kritisieren. [...] Man darf keinen Augenblick vergessen, was Hitler für uns getan hat. [...] alle Maßnahmen seiner Partei haben bis jetzt dazu geführt, das Ansehen unserer Nation auf der ganzen Welt zu erhöhen. “22 - versteht sich von selbst, daß die kollektiven Erwartungen stärkere Legitimation besaßen als ihre eigenen Wertvorstellungen.

Während die privat motivierten Denunzianten den staatlichen Machtapparat für ihre individuellen Interessen nutzten, standen die politisch motivierten Anzeigeerstatter im Dienste des Regimes bzw. seiner Ideologie. Neben systemloyalen Einstellungen, nationalsozialistischen Überzeugungen oder Verdrängungsängsten darf der Aspekt der Pflichterfüllung nicht unterschätzt werden. Die Frau eines alten Parteimitglieds, deren Geschichte im Roman Die Toten bleiben jung dargestellt wird, wartete wochenlang auf Post von ihrem ältesten Sohn. Dann kam Post: Gefallen! „Die Mutter des Gefallenen bekam abends, als Mann und Schwägerin bei ihr saßen, [...] einen hörbaren Ausbruch von Verzweiflung. Sie schrie: ,Daran ist dein Hitler schuld, auf den du immer so stolz warst. '“23 Am nächsten Tag, ihre Schwägerin hatte Anzeige erstattet, wurde die Frau vor das Volksgericht gebracht. „Der Mann bedeutete seiner Frau, sie hätte sich in ihrem Schmerz unüberlegte Worte entschlüpfen lassen, an die sie selbst nicht glaubte. Die Schwägerin hätte ihre Pflicht erfüllen müssen. Was würde aus einem Volk, wenn jeder seiner Verzweiflung nachgäbe?‘ ${ }^{24}$

Die von den NS-Machthabern geförderte Denunziationsbereitschaft der Bevölkerung entwickelte jedoch eine schwer kontrollierbare Eigendynamik, welche die Interessen des Staates zu konterkarieren drohte. Die strafverfolgenden Behörden waren durch die ausgelöste Anzeigenlawine überfordert und nicht unbedingt mit jenen Informationen versehen worden, die sie sich erhofft hatten. Die denunziatorischen Anliegen konzentrierten sich weit mehr auf die Lösung privater Konflikte als auf die Informationen, die den Staat interessierten. Über alle Erwartungen hinaus sind die "Volksgenossen" der Aufforderung zur Anzeige in einem solchen Maße nachgekommen, daß daraus dem Regime selbst erhebliche Probleme erwuchsen.

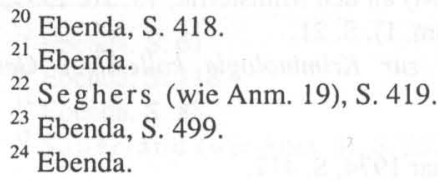


In Verfügungen und Rundschreiben wurde immer wieder „die des deutschen Volkes und des nationalsozialistischen Staates unwürdige Erscheinung des Denunziantentums ${ }^{625}$ kritisiert. Selbst Hitler soll sich darüber ausgelassen haben, „daß wir zur Zeit in einem Meer von Denunziationen und menschlicher Gemeinheit leben“. ${ }^{26}$ Kurz nach der Machtergreifung wurde mit Freiheitsstrafe nicht unter einem Monat Gefängnis oder mit Geldstrafe bedacht, wer einen anderen wider besseres Wissen einer strafbaren Handlung verdächtigte in der Absicht, ein behördliches Verfahren gegen den Betreffenden herbeizuführen. Wurde die falsche Anschuldigung vorsätzlich oder leichtfertig begangen, lag das Strafmaß bei einem Jahr Gefängnis. $^{27}$

Der Erfolg dieser Gesetze war jedoch schon allein deshalb eingeschränkt, weil in der Praxis die wahrheitsgemäße Anzeige einer nach nationalsozialistischem Recht strafbaren Handlung dominierte. Man signalisierte des öfteren den nach den damals geltenden Rechtsnormen strafbaren Umgang mit Juden, Kriegsgefangenen und Zwangsarbeitern oder aber regimekritische Äußerungen, die staatliche Repressionen nach sich zogen. Der politische Denunziant hatte nicht im Sinne, jemanden falsch zu beschuldigen. Seine Anzeigen erschienen in ihrer Rücksichtslosigkeit tödlich wahr.

Mehrere Beispiele derartiger Denunziationsfälle finden wir in Helga Schuberts Geschichtensammlung Judasfrauen. Geschichten nach Akten. Die Autorin hat, vor allem aus Urteilen nach 1945, Geschichten von Denunziantinnen der Nazi-Zeit rekonstruiert und ihr Buch im Jahre 1990 veröffentlicht. Ihr Interesse an dem Stoff lag darin, die Versuchung zum Verrat in einer Gesellschaftsordnung aufzuzeigen, in der es möglich war, private Konflikte mittels Staatsgewalt zu lösen.

In einer dieser zu literarischen Parabeln aufgearbeiteten Geschichten erzählt eine von ihrem Geliebten verschmähte Frau, wie sie zur skrupellosen Denunziantin wurde. Weil der geliebte Mann sie nicht heiraten wollte, erzählte sie ihm, um seine Eifersucht zu provozieren, daß sie als Packerin in einer Fabrik heimlich mancherlei Kontakte (auch nachts) zu den Fremdarbeitern aufrechterhielt.

Er sollte aufwachen. Aber er hat mich angezeigt und mich eines Nachts von der Polizei aus dem Heim holen lassen. [...] Er wußte wie ich, daß manchen Frauen öffentlich die Haare abgeschoren wurden, wenn sie mit Fremdarbeitern ertappt wurden. Mit einem Schild um den Hals mußten sie am Marktplatz stehen: „Ich bin am Ort das größte Schwein, ich laß mich nur mit Polen ein.“ Er wußte, daß diese Männer deshalb aufgehängt werden konnten. Er hat mich und diese Männer der Willkür ausgeliefert, aus Stolz, aus Eigenliebe? Dabei hätte er mich haben können, für ein ganzes Leben. [...] Die Aussicht auf Rache gab mir Kraft in dem Verhör bei der Geheimen Staatspolizei. Und ich zeigte ihn an, noch in der gleichen Nacht. Damals stand auf Abhören feindlicher Sender schon die Todesstrafe. ${ }^{28}$

\footnotetext{
${ }^{25}$ Vgl. das Rundschreiben des Reichsinnenministeriums (RIM) an die Landesregierungen und die Reichsstatthalter, 28. 4. 1934. In: Diewald-Kerkmann (wie Anm. 1), S. 23.

${ }^{26}$ Zit. nach Hinrich Rüping: Denunziation und Strafjustiz im Führerstaat. In: Jerouschek u.a. (wie Anm. 9), S. 128.

${ }^{27}$ Lothar Gruchmann: Justiz im Dritten Reich. 1933 bis 1940. München 1988, S. 835 u. Diewald-Kerkmann (wie Anm. 1), S. 23.

${ }^{28}$ Helga Schubert: Judasfrauen. Geschichten nach Akten. Berlin/Weimar 1990, S. 58.
} 
Am 8. 3. 1940 wies Himmler als Reichsführer der SS und Chef der deutschen Polizei an, daß bei Geschlechtsverkehr zwischen Deutschen und Polen der polnische Partner „Sonderbehandlung“, d.h. Todesstrafe durch Erhängen, erfahren und der deutsche festgenommen werden müsse. Sollte die Bevölkerung den polizeilichen Maßnahmen zuvorkommen und die Delinquenten öffentlich diffamieren, gab es keine Bedenken seitens der Behörden. ${ }^{29}$ Die Opfer auf der deutschen Seite waren bezeichnenderweise nur Frauen, bemerkt Karol Sauerland. Die polnischen Männer wurden gehängt, wenn nicht bewiesen werden konnte, daß sie eindeutschungsfähig seien. ${ }^{30}$

Diese Problematik bildet das zentrale Thema des 1978 erschienenen Dokumentarromans Eine Liebe in Deutschland von Rolf Hochhuth. Seine Protagonisten Pauline Krop und Stasiek Zasada liebten einander ,auf seinen Tod“. 31 Stasiek sollte aushilfsweise in Paulines Laden helfen. Weil gefangenen Ausländern Geschlechtsverkehr mit Reichsdeutschen bei Strafe des Hängens verboten war und weil Elsbeth Schnittgens, Paulines Freundin, den Gemüseladen selbst pachten wollte, kam es zu der folgenschweren Denunziation, die den Polen an den Galgen brachte. Pauline überlebte im Konzentrationslager. Elsbeths Mann hatte noch versucht, seine Gattin von der Anzeige abzuhalten, indem er sie warnte, daß die Betroffenen um ihr Leben kommen könnten. „Ich hab nichts dagegen, daß du ein Geschäft aufmachst, wenn sich eins findet. Ich habe aber keine Lust, eine Frau zu haben, die ihr Geschäft einem Mord verdankt! Du weißt wie ich, der Pole wird umgebracht, wenn wir ihn melden - und sie? Konzentrationslager kriegt die Frau mindestens.“32 Die Antwort fiel lakonisch aus: „Ich sage ja nur - und es ist nun mal verboten! ‘33 Es handele sich um die Wahrheit, beharrte die Frau, und die beiden hätten es sich ja vorher überlegen können.

Die Volksgemeinschafts- und Rassenideologie setzten zwangsläufig eine massive Durchdringung der deutschen Gesellschaft, eine Aufhebung der Grenzen zwischen dem privaten und öffentlichen Leben voraus. Wie wichtig die Beseitigung dieser Grenzen war, machen die gelungenen Versuche der NS-Machthaber deutlich, diese Sphären mit einem dichten Netz von Organisationen, Gliederungen, Einrichtungen und Gruppen zu überziehen. Den Pimpfen, der Hitlerjugend, dem Bund Deutscher Mädel, der Deutschen Arbeitsfront, der NS-Frauenschaft, der Wehrmacht, dem Arbeitsdienst, der Partei, der SA und SS konnte der einzelne nur schwer entfliehen. Im Raster dieser Organisationen wurde er nahezu lückenlos mit Führerbefehlen, internen und militärischen Befehlen, Vorschriften, Sondergesetzen und Erlassen konfrontiert. Diese organisatorische Erfassung erleichterte eine effiziente Ausgrenzung von Gegnern und Andersgesinnten, förderte das Freund-FeindDenken.

${ }^{29}$ S a uerland (wie Anm. 3), S. 42f.

${ }^{30}$ Ebenda, S. 44.

${ }^{31}$ Dieter Lattmann. In: Reinhart Hoffmeister (Hrsg.): Rolf Hochhuth. Dokumente zur politischen Wirkung. München 1980, S. 261.

${ }_{32}$ Rolf Hoch huth: Eine Liebe in Deutschland. Hamburg 1978, S. 101.

${ }^{33}$ Ebenda, S. 98. 
Nach nationalsozialistischer Auffassung vollzog sich das politische Leben allein in der Begegnung von Freund und Feind. Der Feind, behauptete Carl Schmitt, sei letzten Endes jener, der physisch vernichtet werden muß. In diesem Sinne konnte jede menschliche Beziehung eine politische werden, denn jeder Gegner konnte $\mathrm{zu}$ einem physisch $\mathrm{zu}$ vernichtenden öffentlichen Feind werden. ${ }^{34}$ Die Zugehörigkeit zur Volksgemeinschaft artikulierte sich vor allem in der Fähigkeit, die Artverschiedenheit zu erkennen und als Konsequenz dieser Unterscheidung alle diejenigen zu bekämpfen, die als Artfremde und Feinde nicht geduldet werden konnten.

Ernst Lieven, ein fanatischer SS-Funktionär im Roman Die Toten bleiben jung, erwägt die Anzeige seines Vetters Otto. Wegen der Ereignisse um Ernst Röhm, den Chef der SA, beunruhigt, äußerte Otto: „Dir macht es wahrscheinlich nichts aus, wenn Hitler ein paar Grundsätze aufgibt [...]. Aus Grundsätzen machst du dir nichts. Du glaubst nicht einmal an Hitler, du glaubst an Macht, weil etwas davon an dich selbst abfällt. “35 Ernst Lieven sagte am nächsten Tag zu Ottos Schwester: „Mit Otto steht es bereits leider so, daß man vor die Frage gestellt ist: ihm helfen oder ihn anzeigen. [...] Ich kann mir nicht leisten, solche Meinungen auf sich beruhen zu lassen, wie er offenbar das Bedürfnis hat, sie an den Mann zu bringen. [...] Dieser Staat ist mein Staat, ich habe ihm mein Wohl und Wehe anvertraut. Wer zu ihm nein sagt, ist auch mein Feind. “36

Die Erkenntnis, daß Außenstehende, vor allem Angehörige von Fremdgruppen, menschlich in größere Ferne rücken, bildet unter der Bezeichnung der „Distanz“ eine zentrale Kategorie der Kriminologie kollektiver Verbrechen. Für den Hemmungsabbau und die Verminderung von Unrechtsbewußtsein entscheidend ist in erster Linie die Außendistanz gegenüber den Angehörigen anderer Gruppen. „Wir müssen wohl überhaupt von einer von menschlich-sozialer Nähe und Distanz abhängigen Reichweite des Einfühlungs- und Hemmungsvermögens und dadurch relativierten Gewissensreaktionen ausgehen"37, stellt Herbert Jäger fest. Die Außendistanz kann sich dabei zu einer Freund-Feind-Konstellation, verbunden mit Fremdgruppenhaß und aggressivem Verhalten, entwickeln. Demgegenüber sei nichts mehr geeignet, Aggressions- und insbesondere Tötungshemmungen auszulösen, als die innere Nähe bzw. Bekanntschaft mit dem Opfer und die dadurch entstehenden Identifizierungen. Sollten solche Identifizierungsmöglichkeiten fehlen, gilt natürlich das Umgekehrte.

Anna Seghers schildert in ihrem im Exil entstandenen und im Jahre 1942 erschienenen Roman Das siebte Kreuz die Flucht von sieben Häftlingen aus dem Konzentrationslager Westhofen in der Nähe von Frankfurt am Main. Das Phänomen der sozialen Entfernung spielt bei der Verfolgung der Flüchtlinge eine sehr bedeutende Rolle.

\footnotetext{
${ }^{34}$ Vgl. Carl Schmitt: Der Begriff des Politischen. Berlin 1963.

${ }^{35}$ Seghers (wie Anm. 19), S. 354.

${ }^{36}$ Ebenda, S. $355 f$.

${ }^{37}$ Jäger (wie Anm. 17), S. 168.
} 
In einer Kantine unterhalten sich einige Arbeiter über den aus dem Konzentrationslager geflüchteten Georg Heisler. „Warum soll er in seine eigene Stadt flüchten, wo ihn Hunderte Menschen kennen?“" ${ }^{38}$ fragt einer. „Das hat auch Vorteile für den Mann, einen Fremden zeigen die Leute viel leichter an. Stellt euch zum Beispiel mal vor: mich anzeigen! ${ }^{39}$ sagt ein anderer. Ein bei diesem Gespräch anwesender Nazivertrauensmann bemerkt ,in eigentümlich deutlichem Ton“: „Herzensruhig würd ich dich anzeigen, warum denn nicht? Wenn du durch irgend etwas aufhörst, mein Kamerad zu sein, dadurch hörst du doch längst auf, mein Kamerad zu sein, ehe ich aufhöre, deiner zu sein, weil ich dich anzeige. “ ${ }^{40}$

An einer anderen Stelle des Romans Das siebte Kreuz nahmen zwei junge Burschen in einem Büfett eine Mahlzeit ein. „Sie aßen, schwatzten, bis plötzlich der eine abbrach." ${ }^{41}$ Der gesuchte Georg Heisler, der am selben Ort zu Gast war, streifte beim Verlassen des Büfetts

\begin{abstract}
jenen Burschen, der eben bei seinem Anblick zusammengezuckt war. „Hast du denn den gekannt?" fragte der andere. „Fritz“, sagte der erste, ,du kennst ihn ja auch. Hast ihn früher gekannt.“ Der andere sah ihn unsicher an. „Das war sicher Georg“", fuhr der erste fort, ganz offen, außer sich. „Ja, der Heisler, ja, der Geflüchtete.“ Da sagte der andere mit einem halben Lächeln, mit einem schrägen Blick: ,Gott! Du hättest dir was verdienen können.“- „Hätt ich? Hättest du?" - [...] „Nein“, sagte er, ,auch ich hätte es nicht getan." Sie packten ihre Taschen zusammen, früher waren sie ganz gute Freunde gewesen, dann kamen die Jahre, in denen sie nichts Vernünftiges mehr miteinander sprachen aus Angst, sich einander auszuliefern, falls sich der andere verändert hatte. Jetzt hatte sich herausgestellt, daß sie beide die alten geblieben waren. Sie verließen das Büfett in Freundschaft. ${ }^{42}$
\end{abstract}

In einem unmittelbaren Zusammenhang mit der sozialen Distanz stehen die Mechanismen der Entwertung und Deindividuation. Unter Deindividuation ist, nach Herbert Jäger, ein Zustand innerhalb einer sozialen Gruppe zu verstehen, bei dem Gruppenmitglieder andere nicht als Individuen betrachten und dementsprechend auch nicht das Gefühl haben, von anderen als solche gesehen zu werden. Deindividuation führt zu einer Abschwächung innerer Hemmungen mit der Folge, $\mathrm{da} ß$ sich die Gruppenmitglieder wesentlich freier fühlen, Verhaltensweisen $\mathrm{zu}$ zeigen, die sie als einzelne nicht an den Tag legen würden. ${ }^{43}$ Das nationalsozialistische System hat sich diesen Mechanismus in großem Stil nutzbar gemacht, indem es seine Opfer zu nichtmenschlichen Wesen verfremdete und so den Tätern ihre Ausmerzung erleichterte. ${ }^{44}$ Die Entwertung des alten, schwachen, verwerflich reichen, homosexuellen, geschlechtlich allzu freizügigen oder asozialen Opfers ist allerdings ebenso bei alläglichen, nicht unbedingt politisch motivierten Kollektivdelikten festzustellen. Die Mechanismen der Deindividuation, der Entfremdung und Entwertung anderer Menschen können uns auch, natürlich nur zum Teil, erklären,

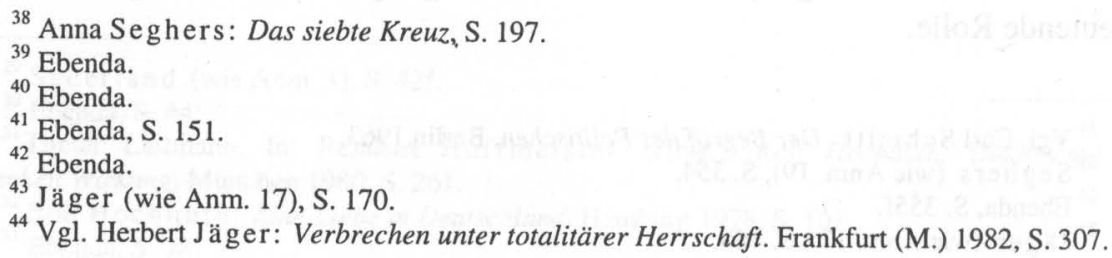


weshalb die Denunzianten weder ein Unrechtsbewußtsein noch irgendwelche Mitleidsregungen mit den Opfern ihrer Anzeigen empfanden.

In der 1943/44 in der Emigration entstandenen Erzählung Der Ausflug der toten Mädchen läßt uns Anna Seghers die drei Schulfreundinnen Marianne, Leni und Netty auf einer Klassenfahrt kennenlernen.

Marianne und Leni und ich, wir hatten alle drei unsere Arme ineinander verschränkt in einer Verbundenheit, die einfach zu der großen Verbundenheit alles Irdischen unter der Sonne gehörte. Marianne hatte noch immer den Kopf an Lenis Kopf gelehnt. Wie konnte dann später ein Betrug, ein Wahn in ihre Gedanken eindringen, daß sie und ihr Mann allein die Liebe zu diesem Land gepachtet hätten und deshalb mit gutem Recht das Mädchen, an das sie sich jetzt lehnte, verachteten und anzeigten. ${ }^{45}$

Der „Wahn“ hat sich eines Tages durch die Entwertung eingeschlichen.

\begin{abstract}
Ehe sie, Leni und ihr Mann, von der Gestapo verhaftet sein würden, sollte Marianne von ihrem [...] Mann Liebig, dem sie auch ewige Treue gelobt hatte, so viel verächtliche Worte über den Mann ihrer Schulfreundin hören, da $B$ ihr selbst bald die Freundschaft mit einem für so verächtlich gehaltenen Mädchen entglitt. Lenis Mann hatte sich mit allen Mitteln gesträubt, in die SA oder SS einzutreten. Mariannes Mann, der stolz auf Rang und Ordnung war, wäre dort in der SS sein Vorgesetzter geworden. Wie er merkte, daß Lenis Mann den von ihm für so ehrenvoll gehaltenen Eintritt verschmähte, machte er die Behörden der kleinen Stadt auf den nachlässigen Untertan aufmerksam. ${ }^{46}$
\end{abstract}

Nachdem Leni und ihr Mann verhaftet waren, erklärten die Gestapobeamten den Nachbarn, das schutzlos zurückgebliebene Kind der Leni gehöre sofort in ein nationalsozialistisches Erziehungsheim. Die Nachbarsfrauen fingen das Kind am Spielplatz ab und hielten es eine Zeitlang versteckt, damit es zu Verwandten des Vaters fahren könnte. Um Reisegeld zu leihen, gingen sie zu Marianne, die sie früher manchmal Arm in Arm mit Leni gesehen hatten. Marianne weigerte sich schroff, diesen Freundschaftsdienst zu leisten. Mit kalten Worten fügte sie hinzu, sie „kümmere sich nicht um ein Mädchen, das irgendwann, irgendwo einmal zufällig in ihre Klasse gegangen sei. Ein jeder Pfennig, an Leni und deren Familie gewandt, sei herausgeworfen, ein Betrug am Staat. [...] Leni samt ihrem Mann seien zu Recht arretiert, weil sie sich gegen Hitler vergangen hätten. “47

Marianne hatte gelernt, den Freund vom Feind gewissenhaft und rücksichtslos zu unterscheiden. Das Gefühl der Zugehörigkeit zu der Gruppe, deren Mitglied Leni nicht werden und deren Ideale sie nicht teilen wollte, verlieh Marianne eine starke, von Gewissensangst und Schuldgefühlen entlastende Kraft. Da ihre Freundin kaum noch einen Wert verkörperte, konnte sie mühelos zu einem konfliktfreien Z1̌l der Aggression werden.

Die vom Staat geförderte Denunziation führte aber, entgegen den Erwartungen der NS-Funktionäre, nicht zum gewünschten Ziel. Die Verfolgung und Beseitigung einzelner politischer Abweichler stabilisierte kaum die politischen Verhältnisse. Die

\footnotetext{
${ }^{45}$ Anna Seghers: Erzählungen 1926-1944. Berlin/Weimar 1981, S. 354f.

${ }^{46}$ Ebenda, S. 347.

${ }^{47}$ Ebenda, S. $336 f$.
} 
Denunziation schürte das Mißtrauen auf allen Ebenen der Gesellschaft, führte zur Erschütterung des Vertrauens, das als Basis familiären wie gesellschaftlichen $\mathrm{Zu}-$ sammenlebens unabdingbar ist. Regeln, welche bestimmte Formen der Ehrlichkeit und Verläßlichkeit im Umgang mit anderen Menschen verlangen, gehören, neben denen, welche z.B. den freien Gebrauch der Gewalt verbieten oder zumindest einschränken, zu den Regeln, die ganz offensichtlich Voraussetzungen des sozialen Lebens sind. „Wenn die Anerkennung dieser elementarsten Regeln in jeder Gruppe von Individuen, die in enger Nachbarschaft miteinander leben, nicht als Selbstverständlichkeit gilt, ist die Bezeichnung der Gruppe als ,Gesellschaft ${ }^{\circ}$ zweifelhaft ${ }^{\star 48}$, bemerkt Herbert Hart. Sicher sei jedenfalls, daß diese Gruppe nicht lange existieren wird.

Tatsächlich befürchteten die Nationalsozialisten, daß das Denunziantentum die Staatsautorität untergrabe, notwendige Loyalitäten bzw. Vertrauensverhältnisse unterminiere und vor allem die Wirtschaft lähme. In einem Erlaß vom 31. Mai 1933 an die Reichsstatthalter und den Preußischen Ministerpräsidenten forderte Hitler, daß die in den letzten Wochen beobachtete Sucht, überall Nachforschungen nach Vergehen aus früheren Zeiten anzustellen und die Schuldigen noch nach Jahren zur Verantwortung zu ziehen, aufhören müsse; dem „,verächtlichen Angebertum“, das sich allenthalben breit gemacht habe, sei entgegenzutreten. Er könne sich des Eindrucks nicht erwehren, daß in vielen Fällen nicht das Verlangen nach Gerechtigkeit der Antrieb sei, führende Männer der Wirtschaft vor Gericht zu ziehen, sondern oft persönliche Aversionen und Rachsucht. Wenn die Staatsanwaltschaft und die Polizei in jedem Falle, der zur Anzeige komme, die vorläufige Festnahme verfüge, werde, so Hitler, ,jenes Angebertum gefördert, das den niederen Instinkten der Menschen und nicht der sittlichen Erhebung des Volkes entspringt“ “ ${ }^{49}$

$\mathrm{Daß}$ die politischen Denunziationen in der NS-Zeit überhaupt eine solche Dynamik entwickeln konnten, hing nicht nur damit zusammen, daß in so zahlreichen Fällen politische Motive lediglich vorgeschoben wurden, um die Denunziation für private Zwecke umzufunktionieren. Der Reiz der politischen Anzeigeerstattung bestand, nach Gisela Diewald-Kerkmann, vor allem darin, daß der Denunziant durch sie unmittelbar an der Macht des Regimes, zumindest als Illusion, partizipieren konnte. $^{50}$

Im Roman Das siebte Kreuz begegnet der Flüchtling Georg Heisler im Sprechzimmer eines jüdischen Arztes dem alten Bauern Binder. Weil der Arzt ihm doch nicht zu helfen vermag, dafür aber zehn Mark abverlangt hatte, wollte der Kranke dem ,Jud [...] seinen Sohn aufs Dach schicken“. ${ }^{51}$ Seitdem er zu Hause angekommen war, ,wälzte er sich auf seinem [...] Sofa - kränker als vorher, glaubte er. Da horchte er auf mit offenem Maul." ${ }^{52}$ Im Radio verlas man eben die Nachricht, daß einige KZ-Häftlinge entflohen wären.

\footnotetext{
${ }^{48}$ Herbert Lionel Adolphus Hart: Der Begriff des Rechts. Frankfurt (M.) 1973, S. $236 \mathrm{f}$.

${ }^{49} \mathrm{Vgl}$. den Erlaß an die Reichsstatthalter und den Preußischen Ministerpräsidenten, 31. 5. 1933.

Zit. nach Diewald-Kerkmann (wie Anm. 1), S. 65.

${ }^{50} \mathrm{Vgl}$. Diewald-Kerkmann (wie Anm. 1), S. 82.

${ }^{51}$ Seghers (wie Anm. 38), S. 72.

${ }^{52}$ Ebenda, S. 108.
} 
Leben und Tod vergaß er, die sich in ihm balgten. Er brüllte die Frau an, ihm rascher in Rock und Schuhe zu helfen. Er ließ den Wagen des Sohnes ankurbeln. Wollte er sich an dem Arzt rächen, [...] an dem Patienten, der mit verbundener Hand gestern ruhig seines Wegs gegangen war, wo ihm doch, wie sich eben herausgestellt hatte, gleichfalls der Tod gebührte? Oder glaubte er einfach, durch ein solches Gebaren sich mit den Lebenden gründlicher zu vermischen ? $^{53}$

Offensichtlich hat ihm der Gedanke, Macht über Leben und Tod anderer Menschen ausüben zu können, ungeahnte Kräfte verliehen. Die Verlockung, am staatlichen Machtapparat auch einmal teilnehmen zu dürfen, erwies sich stärker als alle seine Beschwerden.

Bauer Binder wie auch jeder andere Denunziant konnten unter Ausnutzung der staatlichen Gewalt bzw. durch die Einschaltung des nationalsozialistischen Machtapparates ihre individuellen Interessen unproblematisch durchsetzen oder private Konflikte lösen. Mit dem automatischen Funktionieren einer seelenlosen unbarmherzigen Maschinerie der Jurisdiktion war ohne Zweifel zu rechnen. Nach 1933 entstand in der deutschen Gesellschaft ein Klima, in dem persönliche Anzeigemotive - angefangen von sozialen Ressentiments, Neid, Rache, Zorn bis zu $\mathrm{Ha}$ gegenüber dem Opfer - unter dem „,politischen Mäntelchen“"54 rücksichtslos ausgelebt werden konnten. Hinzufügen muß man dabei, daß die Denunziationsbereitschaft der „Volksgenossen“ vor allem dann forciert wurde, wenn das Regime unter Legitimationszwang geriet. In Zeiten wachsender Mißstimmungen wollte man massiver gegen Kritiker und Andersdenkende vorgehen. Die von Gisela DiewaldKerkmann analysierte zeitliche Verteilung der politischen Denunziationsfälle bestätigt das gesteigerte Unmutspotential bestimmter Gruppen der Gesellschaft in den Jahren 1935/1936 und die äußerst gespannte Lage infolge der militärischen Niederlagen seit $1942 / 1943$. $^{55}$

Während man direkt nach der nationalsozialistischen Machtergreifung eher noch zögerte, Nachbarn, Arbeitskollegen, Verwandte oder ehemalige Freunde anzuzeigen, scheint diese Hemmschwelle im Jahr 1935 überschritten. Ausschlaggebend war nicht nur der intensivierte Kampf gegen die Juden, sondern auch eine gewisse Ernüchterung und Enttäuschung der Bevölkerung.

In der 1937 im Exil entstandenen Szenenfolge Furcht und Elend des Dritten Reiches stellt Bertolt Brecht - sich auf Pressemeldungen und Augenzeugenberichte stützend - Alltagsereignisse in Hitler-Deutschland dar. Die Szene Der alte Kämpfer bietet eine sehr eindrucksvolle Illustration der Atmosphäre dieser Jahre. Der Fleischhändler Lettner, seit 1929 in der nationalsozialistischen Partei, entkam nur deswegen seinen Verfolgern, weil er zu der Zeit, in der man seinen Sohn geholt hat, außerhalb bei einer Viehauktion war. Sonst hätte man auch ihn mitgenommen. Was haben die Lettners verbrochen?

Die Milchhändlerin: Mit dem Fleisch aufgeschlagen. Er bekam nichts mehr herein in der letzten Zeit und mußte die Kunden weggehen lassen. Und da soll er schwarz gekauft haben. Es heißt sogar, beim Juden.

${ }^{53}$ Ebenda.

${ }^{54}$ Das politische Mäntelchen. Polizei warnt Denunzianten. In: Westfälische Neueste Nachrichten v. 31. 10. 1933. Zit. nach Diewald-Kerkmann (wie Anm. 1), S. 82.

${ }^{55}$ Vgl. ebenda, S. 73. 
Der junge Bursche: Und da sollen sie ihn nicht wegholen!

Die Milchhändlerin: Er war immer einer der eifrigsten. Den alten Zeisler von Nummer siebzehn hat er hineingebracht, weil der den Völkischen nicht abonniert hat. Er ist ein alter Kämpfer. ${ }^{56}$

In der Stadt erzählte man sich, die Lettners hätten eine Hypothek auf dem Haus - wobei sie sogar damit gerechnet haben sollen, daß sie von der NS-Regierung gestrichen wird. Der alte Lettner sei früher immer wild geworden, wenn jemand etwas gegen die nationalsozialistische Idee sagte. „Er hat immer von der Idee geredet und gegen den Egoismus von den einzelnen. ${ }^{.57}$ Am Vortage der Verhaftung seines Sohnes hat er viel Krach gemacht, „daß man ihn über den ganzen Platz hat brüllen hören". ${ }^{8}$ Der Alte weigerte sich, die Schinken aus Pappmaché im Schaufenster aufzuhängen, ,die sie ihm gebracht haben. Vorher hat er sie bestellt, weil sie's verlangt haben, weil er eine Woche lang überhaupt nichts ins Schaufenster gehängt hat, nur die Preistafel. [...] Wie sie dann mit dem Pappmachéschinken gekommen sind, hat er gebrüllt, er hängt nichts zum Schein in sein Schaufenster und noch allerhand anderes, was man gar nicht wiederholen kann. Alles gegen die Regierung. "59 Am Ende der Szene erfährt man, daß er sich im Schaufenster seines Ladens erhängt hat, mit einer Preistafel um, auf der zu lesen war: Ich habe Hitler gewählt! Diese Unmutsäußerung mußte aber nicht mehr angezeigt werden. Dem Engagement eines potentiellen Denunzianten ist man "leider" zuvorgekommen.

Hätte der ,alte Kämpfer“ Lettner ein bißchen mehr Geduld mit der NSRegierung und einen etwas stärkeren Glauben an die "Idee" gehabt, hätte er eine ganze Reihe von innen- und außenpolitischen Erfolgen erleben können. Seit 1937 verzeichnete man nämlich einen zahlenmäßigen Rückgang von Anzeigen, der mit dem Wirtschaftsaufschwung, der fast erreichten Vollbeschäftigung und der immer größeren Akzeptanz des Führers in der deutschen Bevölkerung korrelierte. ${ }^{60} 1940$ wurden die Triumphe des Führers euphorisch gefeiert und betont, daß sich die Allgemeinstimmung zu der Auffassung gesteigert habe, „,in der Person des Führers nicht nur den besten Staatsmann, sondern auch den größten Feldherrn der Gegenwart zu besitzen". ${ }^{61}$ In dieser Atmosphäre allgemeiner Hochstimmung und Dankbarkeit konnte man wenig Anlässe zur Denunziation finden. Die Zäsur brachte erst die Jahreswende 1942/43. Die Kämpfe um Stalingrad, die militärische Niederlage im Januar/Februar 1943 und die spürbar werdenden Versorgungsengpässe, die zunehmenden Luftangriffe und Ängste angesichts einer drohenden Kriegsniederlage verschärften die Spannungen in der Gesellschaft. Die Zahl der politischen Denunziationen erhöhte sich schlagartig.

Die im Dritten Reich vorhandene und geförderte Möglichkeit, sich durch besondere Aktivitäten Ansehen zu verschaffen, in Führungspositionen aufzurücken,

${ }^{56}$ Bertolt Brecht: Werke, Bd. 4, Berlin/Weimar/Frankfurt (M.), S. 424.

${ }^{57}$ Ebenda, S. 425.

${ }^{58}$ Ebenda.

${ }^{59}$ Ebenda.

${ }^{60} \mathrm{Vgl}$. Diewald-Kerkmann (wie Anm. 1), S. 73.

${ }^{61}$ Bericht der Gauleitung Westfalen-Nord (Stimmungsmäßiger Überblick über die gesamtpolitische Lage) für den Monat April 1940. Zit. ebenda, S. 74. 
an „großen Aufgaben“ und historischen Ereignissen beteiligt zu sein, oder aber einfach ein paar private Konflikte zu lösen, machte die Gruppenzugehörigkeit emotional äußerst attraktiv. ${ }^{62}$ Wenn ein Volk ,kriegerische Tugenden“ pflegt und der Gewalt einen hohen Wert beimißt, besteht für den einzelnen ein hoher Anreiz, sich seinen Platz in der Gemeinschaft durch Gewaltleistungen zu sichern. Eine besonders erschütternde Variante dieser Gewaltleistungen war die politische Denunziation durch Ehepartner, Familienangehörige oder Kinder. Selbst wenn man berücksichtigt, daß solche Anzeigen relativ selten waren ${ }^{63}$, ist das Ausmaß an Gewissensschwund erschreckend, mit dem Ehepartner sich gegenseitig bzw. Kinder ihren Pfarrer oder Lehrer denunzierten. Wenn Kinder, von der Zugehörigkeit zur Hitlerjugend oder zum Bund Deutscher Mädel fasziniert, ihre Eltern anzeigten, erreichte die durch die Denunziation hervorgerufene Verunsicherung ihren Höhepunkt.

Die von Brecht in der Szene Der Spitzel gestaltete Unterhaltung im Elternhaus eines kleinen Jungen läßt uns diese grauenerregende Atmosphäre einer überall lauernden Gefahr spüren. Weil der Vater im Beisein seines Sohnes einige kritische Bemerkungen über das Braune Haus und die Priesterprozesse fallenließ und weil der Junge kurz danach plötzlich weggegangen ist, gerieten die Eltern in Panik.

Die Frau: Du weiß doch, was sie jetzt immer hineinreden in sie in der HJ. Sie werden doch direkt aufgefordert, daß sie alles melden. [...] Aber er weiß doch, was geschieht, wenn Leute angezeigt werden.

Der Mann: Das ist doch kein Leben mehr! [...] Einen Judas hast du mir geboren! Da sitzt er bei Tisch und horcht, während er die Suppe löffelt, die wir ihm hinstellen, und merkt sich alles, was seine Erzeuger sagen, der Spitzel!

Die Frau: Meinst du, wir sollen irgendwelche Vorbereitungen treffen?

Der Mann: Meinst du, daß sie gleich mitkommen? [...] Verlier nicht die Nerven. Pack mir etwas Wäsche ein. ${ }^{64}$

Allerdings ist ungewiß, ob der Junge (sollte er seinen Vater überhaupt denunziert haben) eher unbewußt der Forderung nachgekommen ist, jede nonkonforme Äußerung zu melden. Aber auch die Möglichkeit, daß er durch die Anzeige Autoritätskonflikte mit dem Vater austragen wolle, wird erwogen:

Die Frau: Der Junge ist ja wirklich eigentlich nicht so, daß er einfach hinläuft und einen anzeigt. Mir ist ganz übel.

Der Mann: Aber rachsüchtig ist er.

Die Frau: Wofür sollte er denn Rache nehmen?

Der Mann: Weiß der Teufel, da gibt's doch immer was. Vielleicht, weil ich ihm seinen Laubfrosch weggenommen habe.

Die Frau: Aber das ist doch schon eine Woche her.

Der Mann: Aber so was merkt er sich. ${ }^{65}$

Nicht nur die Sorge um das Wohlergehen ihrer Kinder bewog viele Eltern zu einem systemkonformen Verhalten, sondern wahrscheinlich auch die Angst vor

\footnotetext{
${ }^{62}$ Vgl. Jäger (wie Anm. 37).

${ }^{63}$ Vgl. Diewald-Kerkmann (wie Anm. 1), S. 144.

${ }^{64}$ B recht (wie Anm. 56), S. $399 f$.

${ }^{65}$ Ebenda, S. $396 f$.
} 
ihnen. Die Kinder waren durchaus bereit, „,ie Henker und Schinder nach Hause zu führen". 66 Ihre Lehrer schwebten natürlich in ähnlicher Gefahr. Im Unterricht konnten doch zahlreiche Autoritätskonflikte entstehen und auf dem recht bequemen Wege einer Anzeige ,gelöst" werden.

Die Frau: Aber in der Schule liegt doch nichts gegen dich vor?

Der Mann: Wie soll ich denn das wissen? Ich bin ja bereit, alles zu lehren, was sie gelehrt haben wollen, aber was wollen sie gelehrt haben? Wenn ich das immer wüßte! [...] Großer Gott! Und da soll man Lehrer sein! Erzieher der Jugend! Furcht habe ich vor ihr! $!^{67}$

Die politische Denunziation entwickelte sich im Dritten Reich zu einer festen Institution, die selbst von Schülern bzw. Konfirmanden für persönliche Zwecke genutzt wurde. Weder private Motive noch eindeutige Verfolgungsinteressen des Anzeigeerstatters hinderten die NSDAP oder die Gestapo, einer Meldung nachzugehen - nicht einmal die Frage, ob ein Kind als zuverlässiger Zeuge gelten kann. Eine strafbare Handlung lag auch dann vor, wenn vertrauliche oder beleidigende Äußerungen politischer Art gegenüber den NS-Führern im engsten Familienkreis gefallen sind.

Einer der Protagonisten der Szenenfolge Furcht und Elend des Dritten Reiches bekennt:

„Ich kenne meine Pflicht als Genosse ${ }^{68}[\ldots]$ Wenn mir meine eigene Mutter was ins Ohr flüstert von wegen Margarinepreisaufschlägen oder so was, gehe ich sofort ins Sturmlokal. Meinen eigenen Bruder lege ich da rein, wenn er über den Freiwilligen Arbeitsdienst meckert. [...], weil wenn wir das nicht so machen, nicht alle gegen unser eigen Fleisch und Blut Stellung nehmen, dann hat auch das Dritte Reich, das wir so über alles schätzen, keinen Bestand. “69

Die Denunziation war im deutschen Strafrecht bis 1945 kein Rechtsbegriff. Erst durch die Gesetzgebung der Alliierten in den Jahren 1945/46 wurde sie als Verbrechen gegen die Menschlichkeit bestimmt. Damit wurde die in den Jahren 1933 bis 1945 begangene Denunziation zu einem Verbrechen nach geltendem Gesetz.

Unter den deutschen Juristen brach darüber ein heftiger Streit aus. Nach Ansicht großer Teile der deutschen Justiz verstieß das Kontrollratsgesetz Nr.10 gegen den rechtsstaatlichen Grundsatz „nulla poena sine lege“ - keine Strafe ohne Gesetz. Damit wurden Tatbestände mit Strafe belegt, die bei ihrer Ausführung straffrei waren. Zahlreiche Rechtswissenschaftler vertraten die Auffassung, daß mit der Ahndung politischer Denunziationen, die während der NS-Zeit weder rechtswidrig waren noch unter Strafandrohung standen, rückwirkend neue Straftatbestände geschaffen wurden. Es sei mit dem Prinzip der Schuld unvereinbar, rückwirkend die Verletzung von Normen unter Strafe zu stellen, die vielleicht als moralische Gebote, nicht aber als Rechtsnormen erkennbar gewesen seien.

\footnotetext{
${ }^{66}$ Ebenda, S. 391.

${ }^{67}$ Ebenda, S. 397-399.

${ }^{68}$ B recht (wie Anm. 56), S. 354f.

${ }^{69}$ Ebenda.
} 
Andere Juristen wiesen ausdrücklich darauf hin, daß eine unbestreitbare ethische Verpflichtung des Staates bestehe, die Denunzianten zu bestrafen. Da das deutsche Strafrecht dazu nicht in allen Fällen ausreiche, habe das rechtsstaatliche Prinzip zurückzustehen hinter der ethischen Notwendigkeit, ein neues, rückwirkendes Ausnahmestrafrecht zu schaffen. Dadurch, daß man die Denunzianten zur Rechenschaft ziehe, würden die Rechtssicherheit und das erschütterte Rechtsbewußtsein wieder hergestellt.

Es ist offensichtlich, daß dieser Streit in erster Linie zwischen den Verfechtern naturrechtlicher Auffassungen und den Rechtspositivisten ausgetragen wurde. Einen erheblichen Einfluß gewann dabei die Position von Gustav Radbruch, der in seinem grundlegenden Aufsatz Gesetzliches Unrecht und übergesetzliches Recht die menschenrechtlichen Grenzen staatlicher Gesetzgebungskompetenz benannte und das Recht vom Nicht-Recht, selbst in Gesetzesform, trennte. Radbruch war der Meinung, daß der Positivismus mit seiner Überzeugung „Gesetz ist Gesetz“ den deutschen Juristenstand wehrlos gemacht hat gegen Gesetze willkürlichen und verbrecherischen Inhalts. Dabei sei der Positivismus gar nicht in der Lage, aus eigener Kraft die Geltung von Gesetzen zu begründen. Er glaube, die Geltung eines Gesetzes schon damit erwiesen $\mathrm{zu}$ haben, daß es die Macht besessen hat, sich durchzusetzen. „Aber auf Macht läßt sich vielleicht ein Müssen, aber niemals ein Sollen und Gelten gründen. " ${ }^{70}$ Weil kein Richter sich auf ein Gesetz, das nicht nur ungerecht, das verbrecherisch ist, berufen und die Rechtsprechung danach handhaben könne, forderte Radbruch, daß ,Richter angeklagt werden [...], die mit den Geboten der Humanität unvereinbare Urteile gesprochen und wegen Nichtigkeiten auf Todesstrafe erkannt haben". 71 Die Rechtsprechung konzentrierte sich jedoch in der Regel auf die Verfolgung von Denunzianten, und die Täter im Machtapparat selbst, z.B. in der Justiz, ließ sie meist ungeschoren. ${ }^{7}$

In der Form jedes Gesetzes kann es nach Radbruch ein „gesetzliches Unrecht“ geben. Nur an dem Maßstab eines übergesetzlichen Rechts könne ermessen werden, was Recht sei, „mag man dieses Recht über allen Gesetzen nun Naturrecht, göttliches Recht oder Vernunftrecht nennen“. ${ }^{73}$ Dieses übergesetzliche Recht könne auch Gesetzesform annehmen. Sein Kennzeichen sei dann die sonst verpönte Rückwirkung des Gesetzes. Deutsches Recht sei in diesen Fällen nicht die Folge des Gesetzes, sondern sein Grund: Das Gesetz formuliere hier nur, was schon vorher Recht war.

Das Jahr 1951 brachte eine Zäsur, als die generelle Ermächtigung zur Aburteilung von Verbrechen gegen die Menschlichkeit nach dem Kontrollratsgesetz Nr. 10 aufgehoben wurde. Das Ergebnis war, daß nicht nur die strafrechtliche Ahndung der NS-Denunziation äußerst schwierig wurde, sondern auch einige Denunzianten, die zuvor wegen Verbrechens gegen die Menschlichkeit zu Frei-

${ }^{70}$ Gustav Radbruch: Rechtsphilosophie, Bd. 3. Hrsg. v. Arthur Kaufmann. Heidelberg 1990, S. 88.

${ }^{71}$ Ebenda, S. 87.

${ }^{72}$ Vgl. Horst Luther: Denunziationen als soziales und strafrechtliches Problem in Deutschland in den Jahren 1945-1990. In: Jerouschek u.a. (wie Anm. 9), S. 261.

${ }^{73}$ Radbruch (wie Anm. 70), S. 96. 
heitsstrafen verurteilt worden waren, in erneuten Verfahren nach den Strafnormen des deutschen StGB straffrei blieben.

Wenn nach einer Revolution oder nach einem größeren Umsturz die Gerichte des neuen Systems ihre Einstellung gegenüber den unmoralischen oder ungerechten Taten definieren müssen, die in legaler Form durch Bürger oder Beamte unter dem vergangenen Regime begangen wurden, kommt es zu großen Kontroversen. Deren Bestrafung mag sozial erwünscht erscheinen, dies aber durch eine rückwirkende Gesetzgebung zu bewirken, indem man kriminalisiert, was vom Recht des früheren Systems zugelassen oder sogar verlangt wurde, scheint zumindest schwierig, moralisch zweifelhaft oder letzten Endes nicht durchführbar. Es ist dann sehr verführerisch zu sagen, bemerkt Herbert Hart, daß Erlasse, die eine Ungerechtigkeit erlauben, nicht als gültig anerkannt werden sollten, weil sie dann automatisch keine Rechtsqualität besäßen. ${ }^{94}$ In dieser Form gewannen die Argumente des Naturrechts im Nachkriegs-Deutschland wieder an Bedeutung - als Antwort auf das akute soziale Problem, das die Verbrechen der NS-Herrschaft hinterlassen hatten. Sollten Menschen, die freiwillig und unentgeltlich dafür sorgten, daß andere wegen ungeheuerlicher NS-Gesetze denunziert, verhaftet und zum Tode verurteilt wurden, zur Verantwortung gezogen werden? War es möglich, sie vor den Gerichten der Nachkriegszeit davon zu überzeugen, daß solche Gesetze, die das Naturrecht verletzen, ungültig seien? Konnten sie wirklich der Auffassung Glauben schenken, daß die Verfolgung der "Verbrechen gegen die Volksgemeinschaft" selbst ein Verbrechen gewesen sei? Wohl kaum.

Der Sinn von Gerechtigkeit, daß es etwas außerhalb des offiziellen Systems gibt, etwas, worauf das Individuum in letzter Instanz seine Gehorsamsprobleme und Gewissenskonflikte projizieren und lösen kann, scheint in Diktaturen bzw. in politisch-revolutionären Umbruchsituationen wenig relevant zu sein. Die Denunziationsbereitschaft dagegen erweist sich als ein erschreckend schnell mobilisierbares Potential.

\footnotetext{
${ }^{74}$ Vgl. Hart (wie Anm. 48), S. 287.
} 